\title{
Wave separation in the trumpet under playing conditions and comparison with time domain finite difference simulation
}

\author{
Jonathan A. Kempa) \\ Department of Music, University of St Andrews, Beethoven Lodge, 65 North Street, St Andrews, \\ Fife KY16 9AJ, United Kingdom \\ Stefan Bilbao \\ Acoustics and Fluid Dynamics Group/Music, University of Edinburgh, Edinburgh EH9 3JZ, \\ United Kingdom \\ James McMaster \\ School of Physics and Astronomy, University of St Andrews, North Haugh, St Andrews, \\ Fife KY16 9SS, United Kingdom \\ Richard A. Smith ${ }^{\text {b) }}$ \\ Richard Smith (M I) Ltd., Cornborough, Sheriff Hutton, York YO60 6RU, United Kingdom
}

(Received 10 January 2013; revised 16 May 2013; accepted 28 May 2013)

\begin{abstract}
Wave separation within a trumpet is presented using three high pressure microphones to measure pressure waves within the curved, constant cross-section tuning slide of the instrument while the instrument was being played by a virtuoso trumpet player. A closer inter-microphone spacing was possible in comparison to previous work through the use of time domain windowing on non-causal transfer functions and performing wave separation in the frequency domain. Time domain plots of the experimental wave separation were then compared to simulations using a physical model based on a time domain finite difference simulation of the trumpet bore coupled to a one mass, two degree of freedom lip model. The time domain and frequency spectra of the measured and synthesized sounds showed a similar profile, with the sound produced by the player showing broader spectral peaks in experimental data. Using a quality factor of 5 for the lip model was found to give greater agreement between the simulated and experimental starting transients in comparison to the values in the range 1-3 often assumed. Deviations in the spectral content and wave shape provide insights into the areas where future research may be directed in improving the accuracy of physical modeling synthesis. (C) 2013 Acoustical Society of America. [http://dx.doi.org/10.1121/1.4812254]
\end{abstract}

PACS number(s): 43.75.Zz, 43.75.Yy, 43.75.Fg, 43.20.Ye [TRM]

Pages: $1395-1406$

\section{INTRODUCTION}

Brass instruments, such as the trumpet, involve a resonator driven by a lip reed, and describing and modeling the sound generating mechanism has been the subject of various articles with many being reviewed by Campbell $^{1}$ and Gough. $^{2}$ The first successful description of the nature of sound production in brass system can be found in Elliott and Bowsher. ${ }^{3}$ Their work focused on the "outward striking" reed model where the lips can be modeled by a single mass with a single degree of freedom which will tend to be forced open to allow air flow into the instrument when the pressure in the mouthpiece is lower than the pressure in the mouth. They also noted the potential for the Bernoulli force to force the lips closed when the flow rate between them was high, an aspect described fully in the related area of vocal sound production by Pelorson et al. ${ }^{4}$ The three basic types of reed or valve behavior described by Fletcher ${ }^{5}$ consist of the "inward striking" model (where the reed closes when the

\footnotetext{
a) Author to whom correspondence should be addressed. Electronic mail: jk50@st-andrews.ac.uk

b) Also at the College of Science and Engineering, University of Edinburgh, Edinburgh, United Kingdom.
}

pressure in the mouthpiece is low, as is the case in the reed in woodwind instruments) and the "sideways striking," sliding door or transverse model (where the Bernoulli force tends to close the lips) in addition to the outward striking model. Both of the later two models are believed to occur in playing brass musical instruments with outward striking model describing oscillation just above, and the sideways striking model describing oscillation just below the resonant frequencies of the air column.,

Various physical models have been created ${ }^{6-10}$ to simulate the sound in brass instruments using parameters approximately matching those of playing conditions. These models tend to treat the response of the trumpet as being characterized by convolution with an impulse response, in which case the pressure is calculated at the mouthpiece position and may be calculated at other points in space using transfer functions. It should be noted that nonlinear propagation influences the radiated sound ${ }^{11}$ and recent work indicates that wall vibrations also have some effect. ${ }^{12}$

A recent physical model by Bilbao ${ }^{13}$ brings the possibility of having a finite difference simulation of the entire instrument, which means that both the pressure and velocity at every position along the instrument bore are calculated as a function of time. This has the advantages of allowing direct 
comparison to the separation of the forward and backward going waves within brass instruments, as will be demonstrated in this paper, and also opens new possibilities in modeling of the waves present while valves are manipulated, as may be demonstrated in a future publication.

Deriving the control parameters from the observed output from an instrument has been possible for simplified brass instrument models. ${ }^{14}$ In analyzing the nature of the lip reed within brass instruments, it is useful to be able to measure the forward and backward going waves within the instrument. A recent paper on wave separation ${ }^{15}$ demonstrates a convenient technique for calibration which relies on calculating the transfer functions for travel between microphones using frequency domain division. Once this has been achieved, wave separation can be carried out using a time domain algorithm ${ }^{15}$ or a frequency domain algorithm. ${ }^{16}$ This has been carried out in brass instruments while they are being played. ${ }^{17,18}$ It should be noted that measuring the pressure in the mouthpiece could give information on the incoming and outgoing waves if the impedance was known accurately there. The lips, however, bulge into the mouthpiece to a varying degree during sounding, turbulence will be present there and the impedance can alter due to the use of valves in general. No theoretical knowledge of the impedance anywhere in the pipe is required for the current work, and there is no requirement for the impedance to remain constant (hence wave separation could be used during valve slurs). The aim here is to compare the results of wave separation in the trumpet while the instrument is being played with physical modeling simulations in order to assess the level of agreement in the time domain waveforms, particularly in the starting transients (without valve transitions). This provides validation of the use of the wave separation technique under playing conditions while also providing information on areas where future development of physical models may be focused.

\section{WAVE SEPARATION}

In order to be able to separate waves traveling to the left and right hand side of a cylindrical section of pipe we need to measure the transfer functions $h_{a b}$ and $h_{b a}$ such that

$$
p_{b}^{+}=p_{a}^{+} \otimes h_{a b}, \quad p_{a}^{-}=p_{b}^{-} \otimes h_{b a}
$$

where $p_{a}^{+}$is the forward going pressure wave at microphone $a$, which is to the left-hand side of microphone $b$ and $\otimes$ denotes convolution.

The transfer function between any two microphones can be measured by coupling a loudspeaker to a cylindrical section of piping as shown in Fig. 1. For a given pair of microphones, we will label the pressure at the left hand microphone as $P_{a}(\omega)$ and the pressure at the right hand microphone as $P_{b}(\omega)$ as expressed in the frequency domain.

As demonstrated in Kemp et al., ${ }^{15}$ frequency domain division can be shown to produce a $z$ domain sequence

$$
\begin{aligned}
\frac{P_{b}(z)}{P_{a}(z)}= & H_{a b}(z)+H_{a b}(z) \times\left[z^{-M_{b}} R_{b}(z)-z^{-M_{a}} R_{a}(z)\right] \\
& \times\left[1-z^{-M_{a}} R_{a}(z)+z^{-2 M_{a}} R_{a}^{2}(z)-\ldots\right],
\end{aligned}
$$

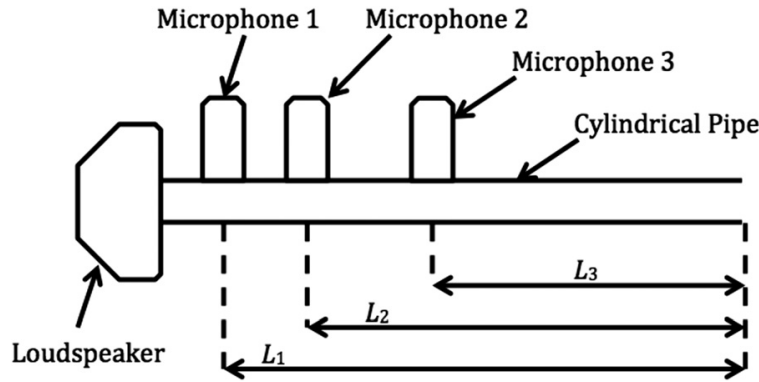

FIG. 1. Calibration apparatus.

where $z^{-M_{a}} R_{a}(z)$ is the multiple reflection sequence beginning with the reflection from the end of the cylindrical pipe to the right of microphone $a$ and $z^{-M_{b}} R_{b}(z)$ is the multiple reflection sequence beginning with the reflection from the end of the cylindrical pipe to the right of microphone $b$. The numbers $M_{a}=2 L_{a} F_{s} / c$ and $M_{b}=2 L_{b} F_{s} / c$ corresponds to the pure delays assuming that the cylindrical pipe extends to a length of $L_{a}$ to the right of microphone $a$, and a distance of $L_{b}$ to the right of microphone $b$, with $c$ being the speed of sound and $F_{s}$ being the sample rate. From Eq. (2) we can deduce that the frequency division of the two microphone signals, when transformed into the time domain, produces an accurate measurement of the transfer function $h_{a b}$ for the first $M_{b}$ samples (with the condition $M_{b}<M_{a}$ following by definition, and this is a correction to the direction of the inequality stated in Ref. 15). Since the resulting frequency domain division features a measurement of $h_{a b}$ which is approximately impulsive assuming that smooth bandwidth limiting is used, ${ }^{15}$ then the $h_{a b}$ may be obtained by time domain windowing the inverse Fourier transform of Eq. (2).

For the experiments performed in this paper, calibration was performed using four repetitions of an exponential sine sweep of length $2^{21}$ samples played into the system end to end with the measured signals during the second, third, and fourth plays at each microphone being averaged and deconvolved from the input sequence to obtain a system impulse response as measured at each microphone. These were then truncated to around a sixtieth of the length (removing artifacts of nonlinearities ${ }^{19}$ ). The frequency domain division of the resulting system impulse response signals where multiplied by a frequency domain low pass filter with a transition band between $14 \mathrm{kHz}$ and $18 \mathrm{kHz}$ before taking the inverse Fourier transform (thus minimizing the effects of noise outside the bandwidth and time domain ripple). Figure 2 shows the result for microphone signals 2 and 1 for the apparatus described in Sec. IV, with different time scales for the same data displayed in the subfigures (and the axis labels omitting the low pass filter for brevity). Note that the decaying series of pulses shown in Fig. 2(a) is caused by end reflections as predicted by Eq. (2). The time domain transfer function $h_{12}$ is then obtained by creating a truncated vector consisting of the initial pulse from Fig. 2(b) with the ripple shown in Fig. 2(c) included on the end of the vector [and the other pulses due to end reflections from Fig. 2(a) removed]. A Fourier transform may then be applied to give an accurate measurement of $H_{a b}(\omega)$ even though the filter is non-causal in the time domain. This means that closer inter-microphone 
(a)

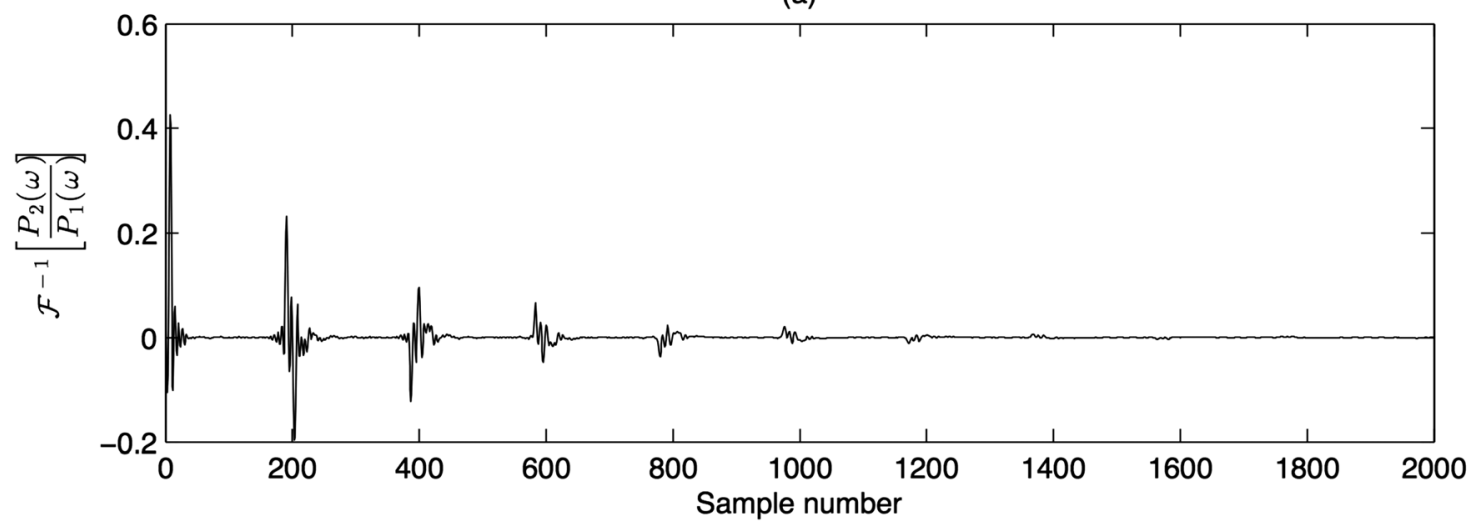

(b)

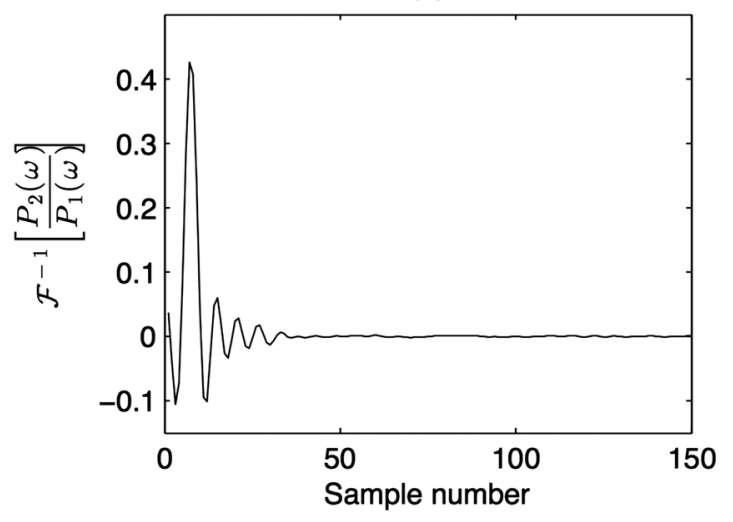

(c)

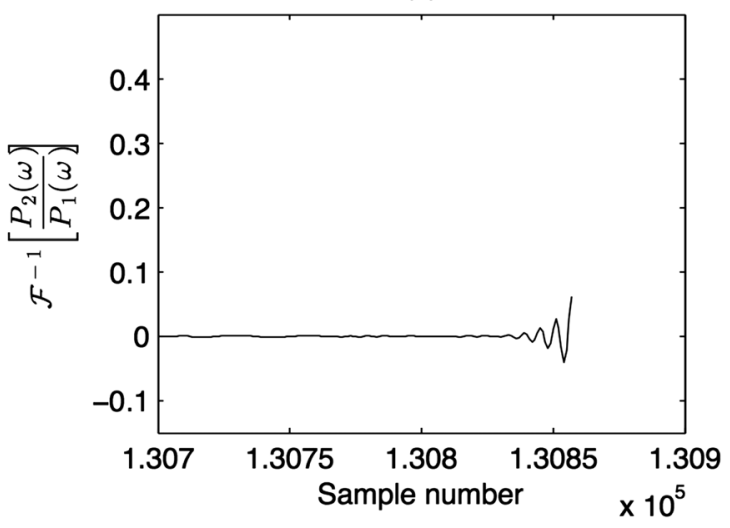

FIG. 2. The result of low pass filtered frequency domain division then taking the inverse Fourier transform for system impulse responses measured at microphones 2 and 1 with a sample rate of $96 \mathrm{kHz}$. (a) The beginning of the time axis shows the transfer function $h_{12}$ is followed by a decaying series. (b) The initial pulse $h_{12}$ occurs at the start of the time axis (c) with ripple extending to the end of the time axis.

spacings are possible in comparison to the previous time domain method from Kemp et al. ${ }^{15}$

In principle, the forward and backward going transfer functions, $h_{a b}$ and $h_{b a}$, might be expected to be the same, but differences in the microphone channel responses means that it is safer to perform separate transfer function measurements for forward and backward going waves using measurements with the loudspeaker situated at either end of the cylindrical pipe as discussed in Kemp et al. ${ }^{15}$ Once this calibration process is done, wave separation can be performed in the time domain as in Kemp et al. ${ }^{15}$ or in the frequency domain as in de Sanctis and van Walstijn. ${ }^{16}$

The Fourier transformed time domain windowed transfer function pulses $H_{a b}(\omega)$ and $H_{b a}(\omega)$, may then be used to perform wave separation experiments by measuring pressures at microphones $a$ and $b$ and obtain the forward going pressure wave at microphone $b$ as ${ }^{15,16}$

$$
P_{b}^{+}(\omega)=\frac{H_{a b}(\omega) P_{a}(\omega)-H_{a b}(\omega) H_{b a}(\omega) P_{b}(\omega)}{1-H_{a b}(\omega) H_{b a}(\omega)} .
$$

This procedure can be carried out with any number of microphones to compensate for the effects of singularities. In particular an inter-microphone distance of $L_{a b}$ will feature singularities at frequencies given by $f_{a b}=n c /\left(2 L_{a b}\right)$ for $n=1,2,3 \ldots$ Since the transfer function between two microphones is approximately impulsive, the singularities will have values of approximately 0 or $\pi$ in the Fourier transform of the corresponding transfer function. When multiple microphones are used the optimum pair of microphones for using at a particular frequency may be chosen by looking for the pair of microphones where the phase angle of the transfer function minimizes the distance from $\pi / 2$ or $-\pi / 2$. If the phase angle in the range $-\pi$ to $\pi$ is given by the $\angle$ operator, then the optimum microphone pair for a particular frequency bin should then be the combination which minimizes the function

$$
\theta_{a b}(\omega)=|| \angle H_{a b}(\omega)\left|-\frac{\pi}{2}\right| .
$$

\section{TIME DOMAIN FINITE DIFFERENCE MODEL}

A model for simulating brass instruments is set out in Bilbao. ${ }^{13}$ In the continuous, linear, lossless case the relationship between acoustic pressure, $p$, and acoustic particle velocity, $v$, in a pipe is described by the equations

$$
\frac{\partial p}{\partial t}+\frac{\rho c^{2}}{S} \frac{\partial}{\partial x}(S v)=0
$$

where $c$ is the speed of sound, $\rho$ the equilibrium density, $S$ is the cross-sectional area which may be a function of the axial distance, $x$, and 


$$
\frac{\partial v}{\partial t}+\frac{1}{\rho} \frac{\partial p}{\partial x}=0
$$

In order to compute the behavior using a time domain finite difference technique, grids must be created with a spacing which depends on the sampling rate, and it is convenient to have the velocity and pressure grids offset as demonstrated in Bilbao ${ }^{13}$ to give

$$
p_{l}^{n}=p_{l}^{n-1}-\frac{\lambda \rho c}{\bar{S}_{l}}\left(S_{l+1 / 2} v_{l+1 / 2}^{n-1 / 2}-S_{l-1 / 2} v_{l-1 / 2}^{n-1 / 2}\right)
$$

and

$$
v_{l+1 / 2}^{n+1 / 2}=v_{l+1 / 2}^{n-1 / 2}-\frac{\lambda}{\rho c}\left(p_{l+1}^{n}-p_{l}^{n}\right),
$$

where the superscript $n$ indicates the (integer) time sample number, the subscript $l$ represents the (integer) spatial sample number and $\lambda=c k / h$ is the Courant number where $k$ is the sampling period (related to the sample rate, $F_{s}$ by $\left.k=1 / F_{s}\right)$ and $h$ is the distance between spatial grid points, $\bar{S}_{l}$ is the cross-sectional area at $x=l h$ and $S_{l+1 / 2}$ is the crosssectional area at $x=\left(l+\frac{1}{2}\right) h$.

Under the special choices of

$$
\bar{S}_{l}=\frac{S_{l+1 / 2}+S_{l-1 / 2}}{2},
$$

then it can be shown that $\lambda \leq 1$ is sufficient for stability; $\lambda$ should be chosen to be as close as possible to unity to minimize the effects of numerical dispersion. ${ }^{20}$

\section{A. Lossy model}

In creating a realistic model of wave propagation in wind instruments it is necessary to include the effects of losses at the walls, and this leads to loss factors which are proportional to the square root of the frequency. ${ }^{21}$ This in turn means that the appropriate equations feature derivatives to the order half ${ }^{13}$

$$
\frac{\partial p}{\partial t}+\frac{\rho c^{2}}{S} \frac{\partial}{\partial x}(S v)+f \frac{\partial^{1 / 2} p}{\partial t^{1 / 2}}=0
$$

where $f$ is a function of the cross-sectional area (and therefore axial distance, $x$, in general)

$$
f(x)=\frac{2(\alpha-1)}{\nu} \sqrt{\frac{\eta \pi}{\rho S(x)}},
$$

where $\alpha$ (the ratio of specific heats), $\nu$ (the square root of the Prandtl number), and $\eta$ (the shear viscosity coefficient) are thermodynamic constants whose values may be found in Keefe, ${ }^{21}$ and

$$
\frac{\partial v}{\partial t}+\frac{1}{\rho} \frac{\partial p}{\partial x}+g \frac{\partial^{1 / 2} v}{\partial t^{1 / 2}}=0
$$

where $g$ is also a function of $x$ in general

$$
g(x)=2 \sqrt{\frac{\eta \pi}{\rho S(x)}} .
$$

Losses may be computed using fractional derivative techniques using a finite impulse response filter to make a fractional difference approximation for an order $q$ as follows

$$
\frac{\partial^{q} p}{\partial t^{q}} \approx \frac{1}{k^{q}} \sum_{m=0}^{\infty} a_{m} p_{l}^{n-m}
$$

where the coefficients follow from the Grünwald-Letnikov definition and may be calculated using a cumulative product using the recursive definition ${ }^{22}$

$$
a_{0}=1, \quad a_{m}=\left(1-\frac{q+1}{m}\right) a_{m-1}, \quad m=1,2,3 \ldots
$$

The loss terms in Eqs. (10) and (12) require order $q=1 / 2$ derivatives, so Eq. (15) generates a decaying series

$$
a=\left[1,-\frac{1}{2},-\frac{1}{8},-\frac{1}{16},-\frac{5}{128},-\frac{32}{1280} \ldots\right],
$$

which will be used in Eq. (14) for the coefficients to be used in representing the loss in Eqs. (10) and (12). This produces a practical implementation as a time domain finite difference scheme for Eq. (10) as

$$
\begin{aligned}
p_{l}^{n}= & \frac{1}{1+\sqrt{k} f_{l}}\left[p_{l}^{n-1}-\frac{\lambda \rho c}{\bar{S}_{l}}\left(S_{l+1 / 2} v_{l+1 / 2}^{n-1 / 2}-S_{l-1 / 2} v_{l-1 / 2}^{n-1 / 2}\right)\right. \\
& \left.-\sqrt{k} f_{l} \sum_{m=1}^{M} a_{m} p_{l}^{n-m}\right]
\end{aligned}
$$

where we include terms generated from Eq. (15) (where it is necessary, in practice, to truncate the infinite series to order $M$ ) beginning with $a_{1}$ (as the term $a_{0}=1$ contributes to the factor on the outside of the brackets). The practical implementation as a time domain finite difference scheme for Eq. (12) is then

$$
\begin{aligned}
v_{l+1 / 2}^{n}= & \frac{1}{1+\sqrt{k} g_{l+1 / 2}}\left[v_{l+1 / 2}^{n-1}-\frac{\lambda}{\rho c}\left(p_{l+1}^{n}-p_{l-1}^{n}\right)\right. \\
& \left.-\sqrt{k} g_{l+1 / 2} \sum_{m=1}^{M} a_{m} v_{l+1 / 2}^{n+1 / 2-m}\right] .
\end{aligned}
$$

\section{Radiation condition}

Equations (17) and (18) make a model for modeling the behavior of the acoustic pressure and particle velocity within a bore of varying cross-section. In order to fully describe the instrument, we also need to specify the behavior at the open end and at the input. Assuming that the open end of the bore occurs at $l=N$ then Eq. (17) requires the value of the velocity $v_{N+1 / 2}^{n-1 / 2}$ at the end of the pipe when the acoustic variables outside the pipe are unknown. This problem is alleviated in Bilbao $^{13}$ by first assuming the radiation impedance follows the unflanged pipe form ${ }^{23,24}$ approximated by a resistance in parallel with an inductance: ${ }^{25}$ 


$$
Z_{r} \approx \frac{\rho c}{S} \frac{j \omega L_{r} R_{r}}{R_{r}+j \omega L_{r}}
$$

with

$$
R_{r}=4\left(0.6133^{2}\right), \quad L_{r}=\frac{0.6133 r}{c},
$$

where $r$ is the radius at the tube end. This is then set equal to the ratio of pressure and volume velocity with the imaginary term removed using

$$
\frac{\partial p}{\partial t}=j \omega p
$$

resulting in the expression ${ }^{13}$

$$
(\rho c) v=\alpha_{1} p+\alpha_{2} m,
$$

where

$$
p=d m / d t
$$

and

$$
\alpha_{1}=\frac{1}{R_{r}}, \quad \alpha_{2}=\frac{1}{L_{r}} .
$$

Equations (22) and (23) give two equations with two unknowns in the finite difference expressions centered on spatial position $N$ and time point $n+1 / 2$ which may be solved to give an inertial term defined recursively

$$
m^{n}=\frac{\left[\frac{2}{k}+\sigma\left(\frac{2 \alpha_{1}}{k}-\alpha_{2}\right)\right] m^{n-1}+2 p_{N}^{n-1}+2 \lambda \rho c v_{N-1 / 2}^{n-1 / 2}}{\frac{2}{k}+\sigma\left(\frac{2 \alpha_{1}}{k}+\alpha_{2}\right)},
$$

with

$$
\sigma=\frac{\lambda S_{N+1 / 2}}{\bar{S}_{N}}
$$

and the update for acoustic pressure at the bell now being possible using

$$
p_{N}^{n}=\frac{2}{k}\left(m^{n}-m^{n-1}\right)-p_{N}^{n-1} .
$$

\section{B. Coupling a lip model}

In order to compute the behavior at the input side we need access to a value for the velocity at the input. In order to simplify the calculation at this point, we will assume that the non-lossy finite difference Eq. (7) holds for the first spatial sample which we will define as being at spatial sample number $l=0$. This then requires access to the velocity value at spatial sample $l=-\frac{1}{2}$ which must be calculated with reference to the position of the lips and pressures surrounding them.

The velocity through the lips depends on the lip displacement, $\xi_{y}$, the width of the lips, $b$, and the difference between the pressure in the mouth cavity, $p_{m}$, and the pressure at the first spatial sample in the bore, $p_{0}$. We will assume that the top and bottom lips are vibrating symmetrically with equal amplitude so that the distance between the lips is given by $2 \xi_{y}$. The velocity may be calculated using the volume velocity according to the Bernoulli equation ${ }^{10}$ divided by the cross-sectional area at $l=-\frac{1}{2}$. Since the start of the bore is actually labeled as occurring at spatial sample $l=-\frac{1}{2}$, the measured cross-sectional area of the trumpet as a function of axial distance was used for $S_{-1 / 2}$ to $S_{N-1 / 2}$ such that $S_{-1 / 2}$ corresponds to the cross-section at the start of the mouthpiece, and values of $\bar{S}_{l}$ for $0<l<N-1$ were obtained using Eq. (9). The value $\bar{S}_{N}$ was then defined as the measured cross-section at the end of the bore at an axial distance of $1.415 \mathrm{~m}$ and the gradient of the cross-section from Eq. (26) requiring $S_{N+1 / 2}$ which was calculated from $S_{N-1 / 2}$ and $\bar{S}_{N}$ using Eq. (9). Using the Bernoulli equation gives the input velocity as

$$
v_{-1 / 2}^{n-1 / 2}=\left(\frac{2 \hat{\xi}_{y} b}{S_{-1 / 2}} \sqrt{\frac{2}{\rho}}\right) \operatorname{sgn}\left(\hat{p}_{m}-\hat{p}_{0}\right) \sqrt{\left|\hat{p}_{m}-\hat{p}_{0}\right|},
$$

where the hat operator is defined as averaging in time, evaluated around $n-\frac{1}{2}$, for example

$$
\hat{\xi}_{y} \equiv \frac{\xi_{y}^{n}+\xi_{y}^{n-1}}{2},
$$

where the superscripts represent (integer) time sample numbers.

Substituting $p_{0}^{n}$ from Eq. (7) produces a term including $v_{-1 / 2}^{n-1 / 2}$ on the right hand side. Squaring and using the quadratic formula to solve gives

$$
v_{-1 / 2}^{n-1 / 2}=\chi\left[\left(\frac{\beta \epsilon S_{-1 / 2}}{2}\right)-\sqrt{\left(\frac{\beta \epsilon S_{-1 / 2}}{2}\right)^{2}+\chi \beta\left(p_{0}^{n-1}-\hat{p}_{m}-\epsilon S_{1 / 2} v_{1 / 2}^{n-1 / 2}\right)}\right],
$$

where

$$
\epsilon=\frac{\lambda \rho c}{2 \bar{S}_{0}}, \quad \beta=\frac{2}{\rho}\left(\frac{2 \hat{\xi}_{y} b}{S_{-1 / 2}}\right)^{2}
$$

and

$$
\chi=\operatorname{sgn}\left(\hat{p}_{m}-\hat{p}_{0}-\epsilon S_{1 / 2} v_{1 / 2}^{n-1 / 2}\right) .
$$

This allows the pressure at the input to be computed using Eq. (7). The lip spacing satisfies a forced, damped harmonic 
motion equation which may be computed in one dimension or two dimensions.

Note that while we have assumed here that the top and bottom lips are vibrating symmetrically with equal amplitude such that the top open area is $2 \hat{\xi}_{y} b$ as in Adachi and Sato, ${ }^{6}$ measurements of human players show that both lips oscillate, with the top lip vibrating with greater amplitude. $^{26,27}$ Another common condition is to assume that the top lip is the only one vibrating, ${ }^{10}$ and this may be obtained in a straightforward manner with the equations presented here by replacing $2 \hat{\xi}_{y} b$ with $\hat{\xi}_{y} b$ in Eqs. (28) and (31).

\section{Two dimensional lip motion}

We will apply the two dimensional model of lip motion by Adachi and Sato ${ }^{6}$ which is formulated as follows

$$
\begin{aligned}
& \frac{\partial^{2} \vec{\xi}}{\partial t^{2}}+\frac{\omega_{0}}{Q} \frac{\partial \vec{\xi}}{\partial t}+\omega_{0}^{2}\left(\vec{\xi}-\vec{\xi}_{\text {equil }}\right) \\
& \quad=\frac{2 b}{m_{\text {lip }}}\left[\left(p_{m}-p_{0}\right)\left(\vec{\xi}-\vec{\xi}_{\text {joint }}\right)^{\perp}+p_{\text {lip }} \vec{e}_{y} d\right]
\end{aligned}
$$

where $m_{\text {lip }}$ is the mass of the lip, $\omega_{0}$ is the natural frequency of the lip (equal to $\kappa / m_{\text {lip }}$ where $\kappa$ is the spring constant), $Q$ is the quality factor of the damped oscillator, $b$ is again the width of the lip (across the mouth perpendicular to both $x$ and $y), d$ is the thickness of the lip in the axial $(x)$ direction, $\vec{\xi}$ is a vector of the two dimensional displacement of the lip whose first entry is the position in the axial direction (along $x$ ) and whose second entry is the position in the direction transverse to the flow (along $y$ ). The equilibrium position (in the absence of differences in air pressure) is given by $\vec{\xi}_{\text {equil }}$ and the position of the top of the lip is given by $\vec{\xi}_{\text {joint }}$ where the perpendicular operator, $\perp$, is defined by $\vec{A}^{\perp}=\left(A_{x}, A_{y}\right)^{\perp}$ $=\left(-A_{y}, A_{x}\right)$. The pressure in the mouth is given by $p_{m}$ and the pressure in the mouthpiece by $p_{0}$. The pressure beneath the lip, $p_{\text {lip }}$, may be approximated using $p_{\text {lip }} \approx p_{0}$ although a very slight correction is to use continuity as suggested by Adachi and Sato ${ }^{6}$

$$
p_{\text {lip }}=p_{0}-\rho v^{2}\left(\frac{S_{\text {cup }}}{S_{\text {lip }}}-1\right)
$$

where $v$ is the acoustic particle velocity in the mouthpiece, $S_{l i p}$ is the area of the lip opening (given by $2 b \hat{\xi}_{y}$ if two lips are assumed to be vibrating), and $S_{\text {cup }}$ is the area at the beginning of the mouthpiece. Producing a finite difference equation centered on time sample number $n-1 / 2$ produces

$$
p_{\text {lip }}^{n}=-p_{\text {lip }}^{n-1}+p_{0}^{n}+p_{0}^{n-1}-2 \rho\left(v_{-1 / 2}^{n-1 / 2}\right)^{2}\left[\frac{\bar{S}(0)}{2 \hat{\xi}_{y} b}-1\right]
$$

where care must be taken to replace the term $p_{0}^{n-1}$ with zero if $\hat{x}$ was less than or equal to zero at the previous time step in order to ensure stability.

Turning the model from Eq. (33) into a finite difference update scheme produces

$$
\left(\begin{array}{l}
\xi_{x}^{n+1} \\
\xi_{y}^{n+1}
\end{array}\right)=\gamma_{1}\left(\begin{array}{c}
\xi_{x}^{n} \\
\xi_{y}^{n}
\end{array}\right)-\gamma_{2}\left(\begin{array}{c}
\xi_{x}^{n-1} \\
\xi_{y}^{n-1}
\end{array}\right)+\gamma_{3}\left(\begin{array}{c}
\omega_{0}^{2} \xi_{\text {equil }}+\left(2 b / m_{\text {lip }}\right) \Delta p\left(\xi_{\text {joint }}-\xi_{y}^{n}\right) \\
\omega_{0}^{2} \xi_{\text {equil }}+\left(2 b / m_{\text {lip }}\right)\left[\Delta p\left(\xi_{x}^{n}-\xi_{\text {joint }}\right)+p_{\text {lip }} d\right]
\end{array}\right)
$$

where $\Delta p=p_{m}-p_{0}$ and

$$
\begin{aligned}
& \gamma_{1}=\frac{2-\omega_{0}^{2} k^{2}}{1+\omega_{0} k / 2 Q}, \quad \gamma_{2}=\frac{1-\omega_{0} k / 2 Q}{1+\omega_{0} k / 2 Q}, \\
& \gamma_{3}=\frac{k^{2}}{1+\omega_{0} k / 2 Q},
\end{aligned}
$$

where $k=1 / F_{s}$ is again the sample period arising from taking finite difference approximations to the derivatives.

\section{Closed lip condition}

If the lip is closed at a particular time point $(\hat{x} \leq 0)$, then the input velocity, $v_{-1 / 2}^{n-1 / 2}$, [usually provided in Eq. (30)] is set to 0 and damping must be increased. We approximate the change in the forced damped harmonic oscillator behavior by removing the Bernoulli force beneath the lip and by making the spring constant a factor of 3 greater and the damping term, $\omega_{0} / Q$, a factor of 4 greater (as in Vergez and Rodet $\left.^{10}\right)$. This may be implemented in Eq. (36) by temporarily setting $d=0$, and changing the $\gamma$ values from Eq. (37) to have $\omega_{0}$ a factor of $\sqrt{3}$ times bigger and setting $Q$ to be multiplied by a factor of $0.25 \sqrt{3}$. The closure dependent changes in spring force and damping were only implemented for the $y$ direction components because this is the dimension along which the lips collide.

\section{E. Model parameters}

Parameters used in the physical model, including the frequency dependent mass, are similar to those used by Adachi and Sato, ${ }^{6}$ with a $Q$ factor of 5 taken as in Saneyoshi et al. ${ }^{28}$ These parameters are described in Table I. Empirical adjustment of the parameters was used to provide a reasonable degree of agreement between the phase and relative amplitude envelopes of the physical modeling simulation and the experimental results obtained with the human player. It may be noted that the time taken for the starting transient to give way to the steady state oscillation decreases with increasing lip $Q$ factor as demonstrated by $\mathrm{Keefe}^{7}$ and the 
TABLE I. Lip model parameters.

\begin{tabular}{|c|c|c|}
\hline Parameter & Sounding Pitch $\mathrm{Bb}_{4}$ & Sounding Pitch $\mathrm{Bb}_{5}$ \\
\hline$b$ & $7.0 \times 10^{-3} \mathrm{~m}$ & $7.0 \times 10^{-3} \mathrm{~m}$ \\
\hline$d$ & $2.0 \times 10^{-3} \mathrm{~m}$ & $2.0 \times 10^{-3} \mathrm{~m}$ \\
\hline$\left(\xi_{\text {joint }_{x}}, \xi_{\text {joint }_{y}}\right)$ & $\left(0,4.0 \times 10^{-3}\right) \mathrm{m}$ & $\left(0,4.0 \times 10^{-3}\right) \mathrm{m}$ \\
\hline$\xi_{\text {equil }}$ & 0 to $1 \mathrm{~mm}$ between 10 and $20 \mathrm{~ms}$ & 0 to $2 \mathrm{~mm}$ between 10 and $20 \mathrm{~ms}$ \\
\hline$\xi_{\text {equil }}$ & $0 \mathrm{~m}$ & $0 \mathrm{~m}$ \\
\hline$Q$ & 5.0 & 5.0 \\
\hline$m_{\text {lip }}$ & $1.5 /\left(2 \pi \omega_{0}\right)$ & $1.5 /\left(2 \pi \omega_{0}\right)$ \\
\hline$p_{m}$ & 0 to $6.0 \mathrm{kPa}$ over first $10 \mathrm{~ms}$ & 0 to $10.0 \mathrm{kPa}$ over first $10 \mathrm{~ms}$ \\
\hline$f_{0}=\omega_{0} /(2 \pi)$ & $400 \mathrm{~Hz}$ to $450 \mathrm{~Hz}$ between $25 \mathrm{~ms}$ and $35 \mathrm{~ms}$ & $890 \mathrm{~Hz}$ \\
\hline
\end{tabular}

value $Q=5$ gave a better fit between the simulated and experimentally measured transients than the value of 3 used by Adachi and Sato. ${ }^{6}$ During this process three of the physical model parameters were made time varying during the starting transient. These were the mouth pressure (which featured a linear ramp from $0 \mathrm{~Pa}$ to full pressure over the first $10 \mathrm{~ms}$ ), the natural frequency of the lip (which was initially held at a lower frequency value then given a linear ramp to a final value during the starting transient in the case of the $\mathrm{Bb}_{4}$ simulation, but held constant for the $\mathrm{Bb}_{5}$ simulation), and the horizontal equilibrium, $\xi_{\text {equil }_{x}}$, (which was initially held at 0 for $10 \mathrm{~ms}$ and then given a linear ramp to full value over the following $10 \mathrm{~ms}$ ).

The model was run assuming lossy propagation with an order of $M=200$ used in Eq. (17) and (18) and a sample rate of $192 \mathrm{kHz}$. This requires significant storage and means the model runs much slower than real time in the current MATLAB implementation (in spite of using circular buffer techniques). The input impedance implied by the model was calculated by replacing the lips with an impulsive volume velocity source and running the time domain finite difference model, storing the pressure at the mouthpiece and taking the Fourier transform. This was found to show reasonable $( \pm 1 \mathrm{~dB})$ agreement with the input impedance calculated by using the impedance projection techniques. ${ }^{29}$ Future work will investigate techniques that minimize the computation cost and maximize the accuracy of the loss and radiation models.

\section{F. The mouthpiece and volume excluded by the lips}

It is well known that the volume of the mouthpiece is important in determining the playability of the high and low ranges of brass musical instruments, and recent work has proved that the effect of the lips bulging to exclude part of the air volume in the mouthpiece is an important factor. ${ }^{30}$ This was achieved using acoustic measurement of the reflection from lips being held closed against a mouthpiece rim. The results demonstrate that the volume excluded by the lips for one player was $0.8 \mathrm{~cm}^{3}$ and further testing on another player obtained a figure of $0.5 \mathrm{~cm}^{3}$. While the trumpet used by the human player in the experimental wave separation results was played using a standard mouthpiece design (model 7C with cup volume to narrowest point of $1.2 \pm 0.1$ $\mathrm{cm}^{3}$ ), the bore used for the simulations was modified at the mouthpiece to reduce the volume by $0.5 \mathrm{~cm}^{3}$ to give a the cup volume of $0.7 \mathrm{~cm}^{3}$ (again calculated to the narrowest point). It should be noted that mouthpieces with larger cup volumes of around $1.7 \mathrm{~cm}^{3}$ are used frequently by orchestral players, and if no volume correction was implemented for such cup volumes then the physical model starting transient for the $\mathrm{Bb}_{5}$ note was significantly more gradual. This reinforces the importance of taking into account lip protrusion when considering the input impedance and playability of brass instruments and when designing physical models.

\section{RESULTS}

Wave separation results were performed using three high pressure Endevco model 8507C-1 microphones incorporated into a trumpet built by Smith-Watkins to feature a specially commissioned tuning slide section. This tuning slide was built of two removable pieces, one featuring a $90^{\circ}$ bend with a water-key to ensure that the trumpet matched the standard instrument in its internal profile, and the other with a constant cross-section through a $90^{\circ}$ bend and allowing three flush mounted microphones with spacings $L_{12}=$ $23.7 \mathrm{~mm}$ and $L_{23}=13.8 \mathrm{~mm}$ (giving $L_{13}=37.5 \mathrm{~mm}$ ) whose signals were sampled by a Focusrite Saffire Pro 10 soundcard. Calibration was performed by first removing the section of tubing containing the microphones from the trumpet, and coupling it to a JBL 2426J compression driver loudspeaker on one end. A $30 \mathrm{~cm}$ section of cylindrical pipe (with a small piece of foam on the end to minimize pressure nodes within the pipe) was attached to the other end to allow for sufficient number of samples of accuracy in the measurement of the transfer functions using an exponential sine sweep as excitation and utilizing frequency domain division of the microphone signals followed by time domain windowing as described in Sec. II.

Once calibration was performed, short notes were played on the trumpet by the late virtuoso Derek Watkins (in November 2010). The player was instructed to keep the notes short in order to prevent the speed of sound changing significantly during the performance. Once a selection of notes had been performed another calibration measurement was performed to obtain the transfer functions after any small change in the speed of sound. Removing the custom tuning slide for calibration will imply some unavoidable error due to air circulation, but calibrations obtained before 


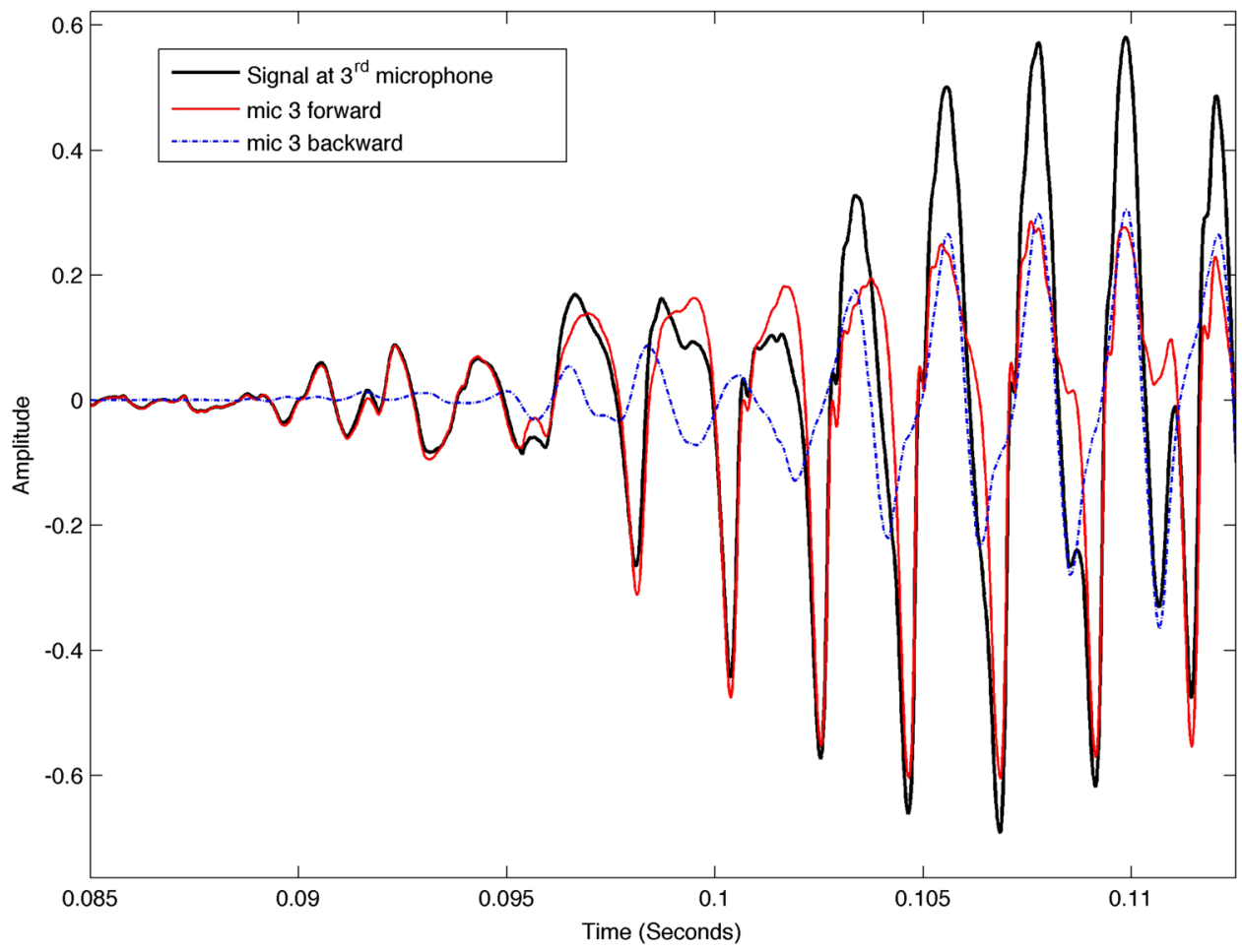

FIG. 3. (Color online) Experiment: Wave separation for the starting transient of the note concert $\mathrm{Bb}_{4}$ on the trumpet played by DW (as obtained $45 \mathrm{~cm}$ from the mouthpiece).

and after the player performing on the instrument were found to show no noticeable differences (due to the player only playing a few short notes). Wave separation was calculated as discussed in Sec. II for the third microphone in the custom tuning slide at a point $45 \mathrm{~cm}$ along the bore from the mouthpiece.

The results of wave separation are shown in Fig. 3 for the note $\mathrm{Bb}_{4}$ and in Fig. 4 for the note $\mathrm{Bb}_{5}$. Time domain finite difference simulations with forward and backward going pressure waves extracted at the corresponding spatial point for a distance of $45 \mathrm{~cm}$ from the mouthpiece are shown for the same notes in Figs. 5 and 6 (with the results high pass filtered with a cut-off frequency of $100 \mathrm{~Hz}$ in order to allow direct comparison with the experimental data (which are insensitive to components below $20 \mathrm{~Hz}$ ). Each plot begins with the measured pressure and forward going wave coinciding. Reflections appear in the form of a backward going pressure wave after a time delay due to the round trip travel time to the bell. These reflections are smoother (more sinusoidal) than the forward going wave due to the low pass filter

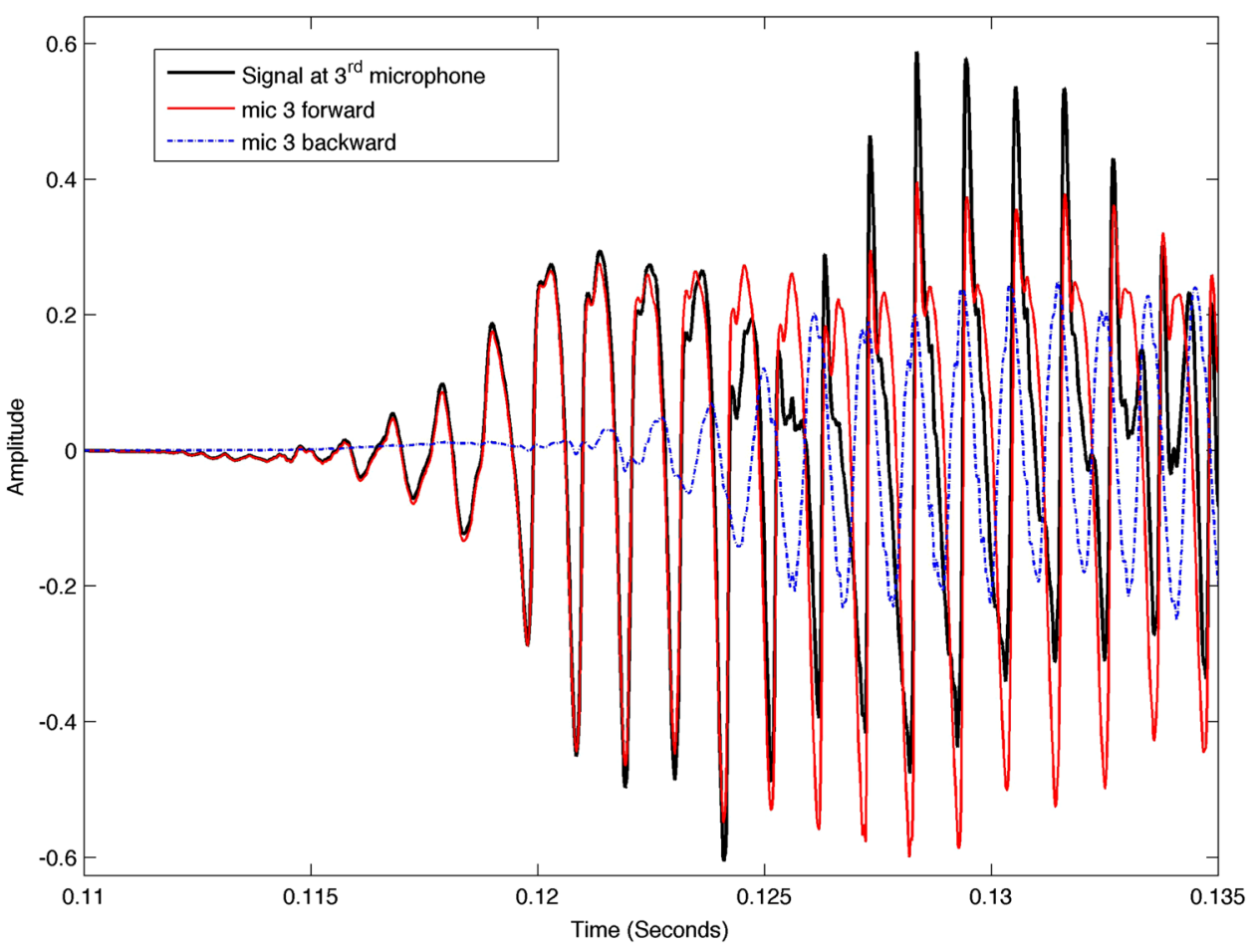

FIG. 4. (Color online) Experiment: Wave separation for the starting transient of the note concert $\mathrm{Bb}_{5}$ on the trumpet played by DW (as obtained $45 \mathrm{~cm}$ from the mouthpiece). 


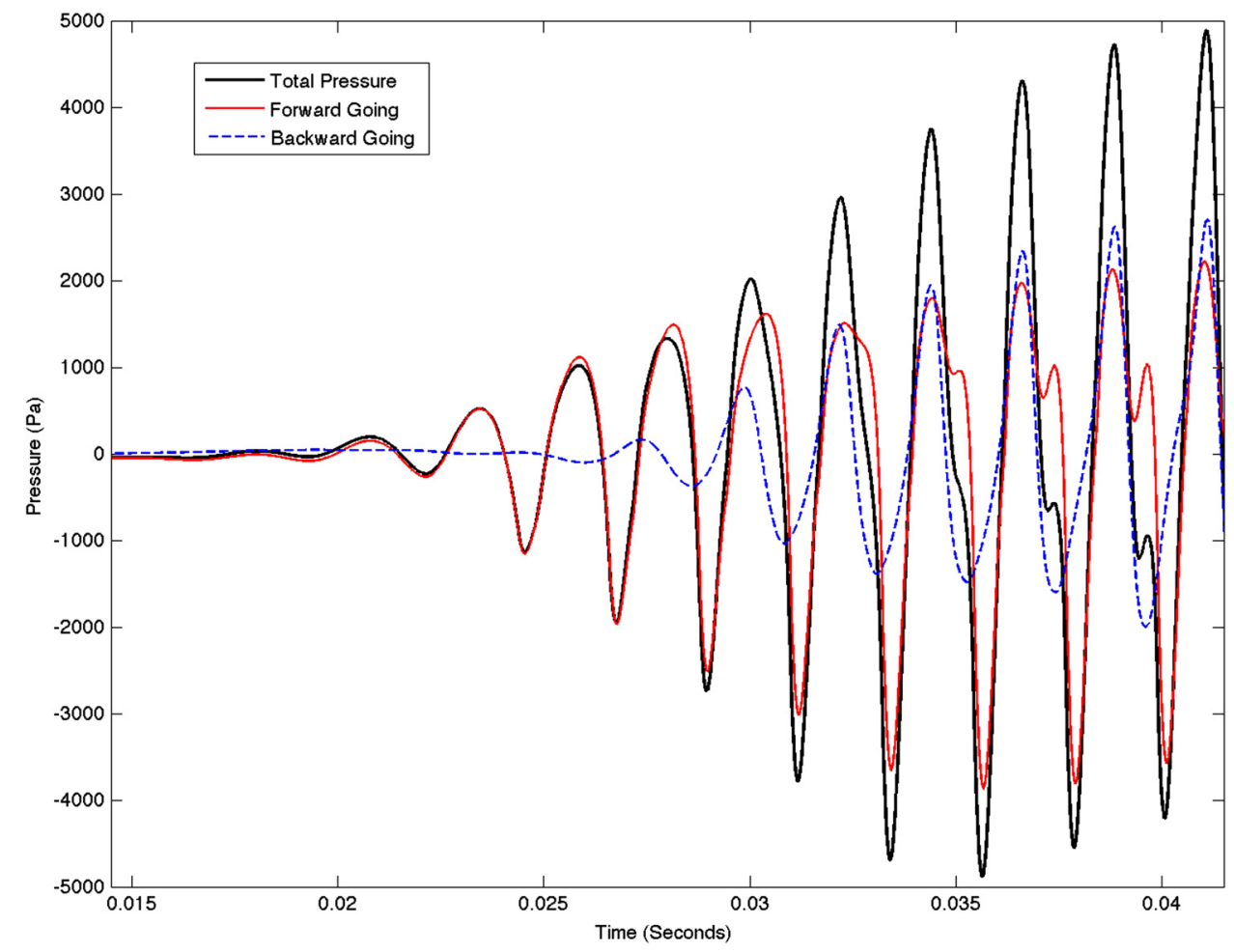

FIG. 5. (Color online) Simulation: Wave separation for the starting transient of the note concert $\mathrm{Bb}_{4}$ on the trumpet physical model (as obtained $45 \mathrm{~cm}$ from the mouthpiece).

nature of the bell reflectance. The overall amplitude envelope profile of transient for the forward and backward going waves in the experimental and simulated data agree well.

In order to check the validity of the experimental wave separation data, the reflectance magnitude at the position of the third microphone was calculated by dividing the measured backward going pressure wave by the forward going pressure wave in the frequency domain for the entire note length (approximately $0.6 \mathrm{~s}$ ). This process is complicated by the fact that these measurements, being taken while the instrument was being played, are strongly periodic and only a small amount of energy (due to the starting transient in particular) is present in between the harmonics. The reflectance calculation therefore shows noisy results between these harmonics. For this reason the data was analyzed in $50 \mathrm{~Hz}$ sections and only the data point containing the strongest signal within that range was used for plotting the reflectance seen in Fig. 7 for the note $\mathrm{Bb}_{4}$ and in Fig. 8 for the note $\mathrm{Bb}_{5}$. The

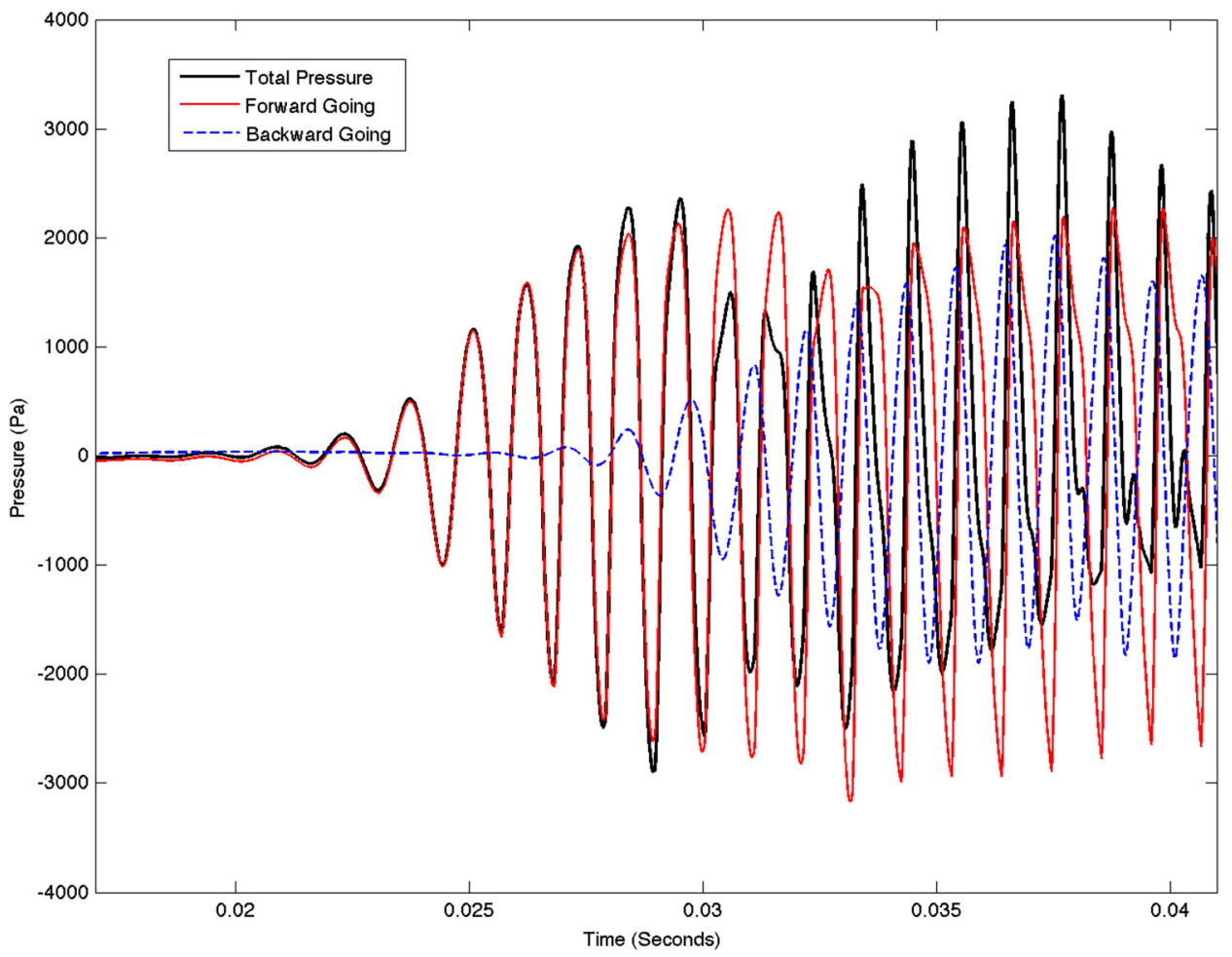

FIG. 6. (Color online) Simulation: Wave separation for the starting transient of the note concert $\mathrm{Bb}_{5}$ on the trumpet physical model (as obtained $45 \mathrm{~cm}$ from the mouthpiece). 
results show good agreement between the reflectance calculated from the experiment wave separation data and that calculated at the position $45 \mathrm{~cm}$ into the trumpet bore using multimodal theory, ${ }^{29}$ particularly at the harmonics seen in the corresponding spectrum plot.

Spectrum plots are also given in the lower plots within Figs. 7 and 8, showing the spectra for the pressure taken $45 \mathrm{~cm}$ from the mouthpiece. The "Simulation" data shown on the plots consists of the spectra of the total pressure for an entire note (taken at a distance of $45 \mathrm{~cm}$ from the mouthpiece as before) where the time domain finite difference simulation was run for $0.75 \mathrm{~s}$, with the mouth pressure linearly decaying to 0 between $0.5 \mathrm{~s}$ and $0.6 \mathrm{~s}$ in order to approximate the end transient created by the player. In each case the sound pressure level was normalized to the maximum (fundamental) peak. The spectrum produced by the physical model for the note $\mathrm{Bb}_{4}$ shows a broadly similar profile to that of the physical model, but the noise floor and excitation of frequencies either side of the resonant frequency are significantly more accentuated in the note produced by the player, as can be seen in Fig. 7. A larger difference between the spectral content of the physical model and human player for the note $\mathrm{Bb}_{5}$ can be observed in Fig. 8 .

Differences between the experimental and simulated data are clear in the initial $5 \mathrm{~ms}$ of the transients in both Figs. 5 and 6 with the experimental data showing a very brief low amplitude oscillation within the experimental data at a higher frequency than the final steady state note. This behavior is not accounted for in the physical model simulation and may be inconsistent with the behavior expected from a one mass model, even with two degrees of freedom. The use of a two mass model allows for the Bernoulli force to be significantly different during the opening and closing phases of the vibration due to the downstream mass lagging behind the upstream mass and future work may focus on whether this can produce the desired behavior.

There is also a clear difference in the shape of the waveforms during both the transient and steady state behaviors in that the forward going pressure wave is more sinusoidal in the simulation and the experimental data being closer to a triangle waveform during the starting transient and showing a sharper negative pulse waveform in the steady state behavior. One possible cause of a significantly non-sinusoidal waveform in the lower amplitude region would be if there was a significant force due to adhesion of the lips which could provide discontinuous acceleration during the opening and closing movements. Future work should include experiments and numerical simulation in this area in order to see if some of the differences between theory and experiment shown in this paper may be explained in this way.

It is likely that the higher amplitude steady state waves show non-linear wave propagation in the instrument bore. The wave separation technique works by assuming linear transfer between microphones and nonlinear transfer between microphones might be expected to yield unexpectedly high harmonic content at microphones more distant from the mouthpiece in comparison to the signal measured closer to the mouthpiece, causing false backward going high frequency harmonic content in the deduced backward going wave. In fact the small inter microphone spacing (maximum
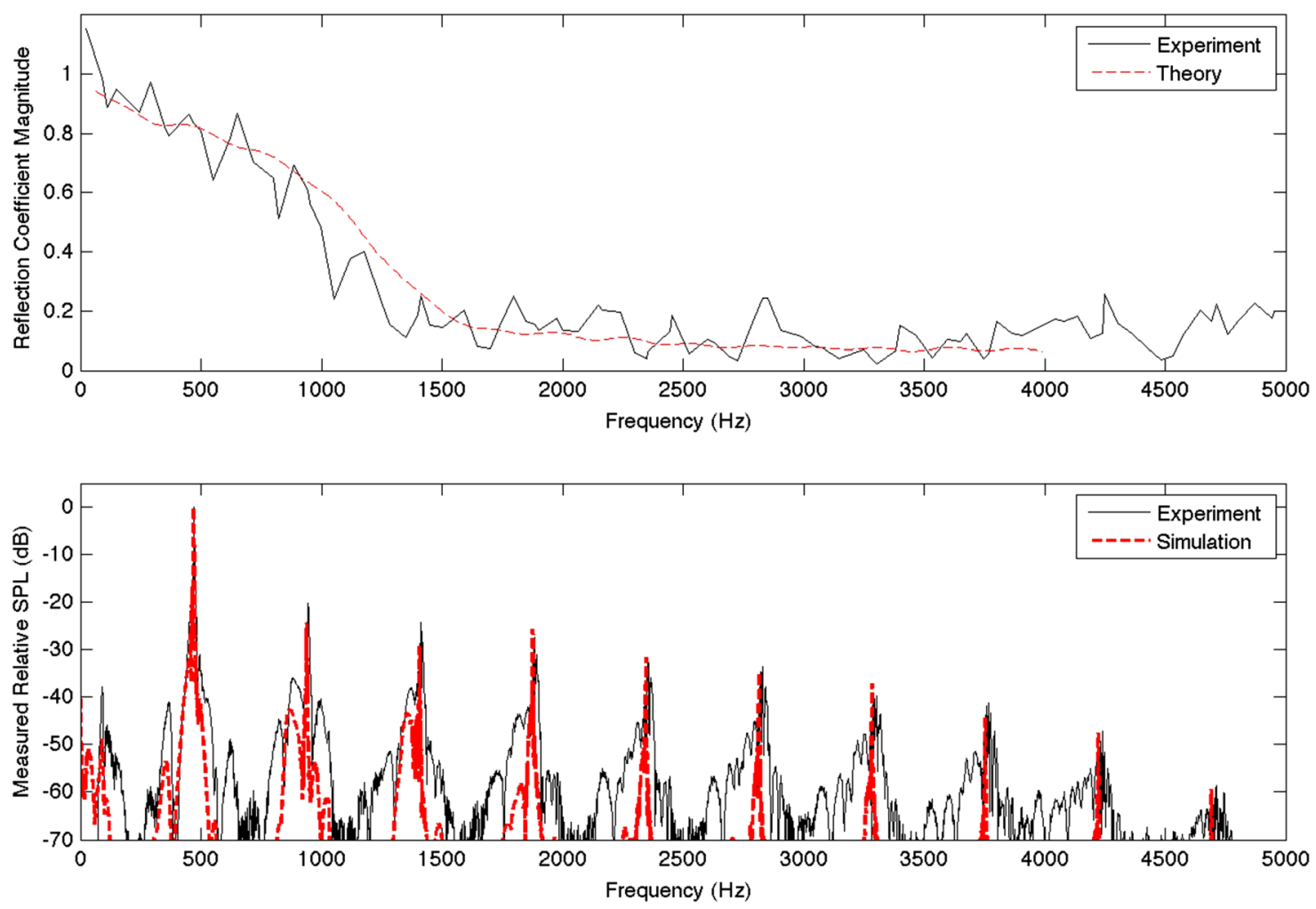

FIG. 7. (Color online) (Top) Reflectance magnitude calculated using wave separation data ( $45 \mathrm{~cm}$ from the mouthpiece) for the note concert $\mathrm{Bb} 4$ on the trumpet played by DW (Experiment) and that obtained by multimodal decomposition (Theory); (Bottom) spectrum of the same note played by DW (Experiment) and the spectrum for the note $\mathrm{Bb}_{4}$ obtained from the physical model (Simulation). 

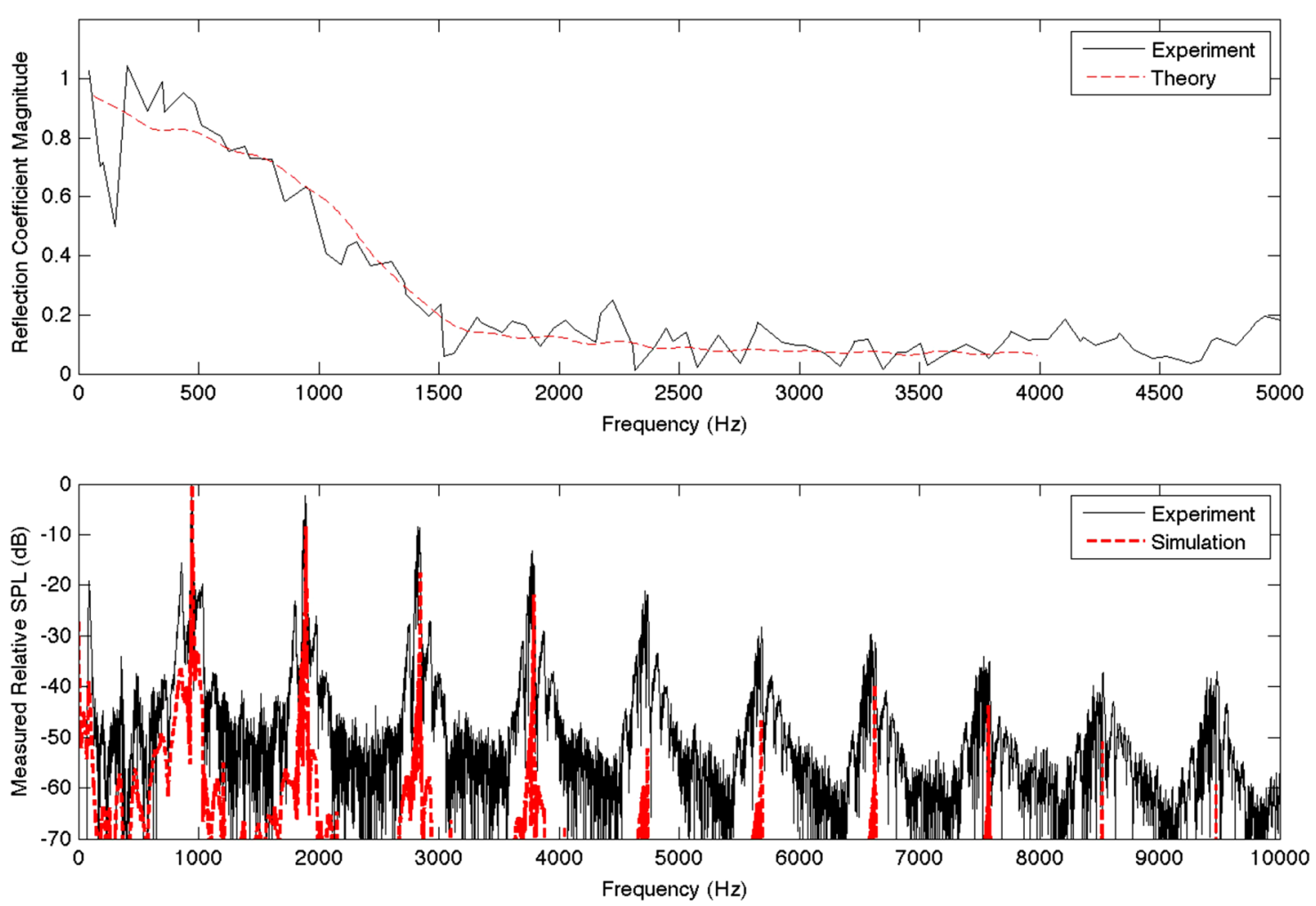

FIG. 8. (Color online) (Top) Reflectance magnitude calculated using wave separation data ( $45 \mathrm{~cm}$ from the mouthpiece) for the note concert $\mathrm{Bb} \mathrm{b}_{5}$ on the trumpet played by DW (Experiment) and that obtained by multimodal decomposition (Theory); (Bottom) Spectrum of the same note played by DW (Experiment) and the spectrum for the note $\mathrm{Bb}_{5}$ obtained from the physical model (Simulation).

microphone spacing $L_{13}=37.5 \mathrm{~mm}$ ) in comparison to the mouthpiece to microphone distance $(450 \mathrm{~mm})$ means that the transfer between microphones is largely linear over this scale, as can be seen from the lack of large spurious peaks in the reflectance magnitude in Figs. 7 and 8. Including nonlinear propagation in the simulations (in the time domain finite difference technique) has been set out by Bilbao ${ }^{13}$ and future work may include addressing the numerical instability that develops with shockwave formation.

\section{CONCLUSIONS}

Wave separation results are presented for measurements taken within the trumpet while the instrument was being played by a virtuoso human player. Simulations, using a time domain finite difference technique coupled to a one mass, two degrees of freedom lip model, demonstrate the success of the wave separation technique in calculating the forward going and backward going waves from pressure measurements using both time domain and frequency domain plots. Areas where the realism of the current lip model and wave propagation assumptions could be improved are also clear and are helpful in considering areas of future physical modeling research. The demonstration of wave separation within the trumpet under playing conditions also means that real-time applications, such as an instrument that may be played by buzzing the lips but with a reflectance under active control using a loudspeaker to provide backward going waves for instance, may be a possibility. This would require using the time domain formulation of wave separation for low latency, adaptive control of the inter- microphone transfer functions due to the changing speed of sound and challenges remain with coping with mean flow, moisture build up, non-linear behavior and the high pressure levels required from the driver.

\section{ACKNOWLEDGMENTS}

We would like to acknowledge the School of Physics and Astronomy at the University of St Andrews for the Student Staff Council Summer Vacation Award that funded James McMaster's contribution to this project. The authors are also very grateful for the help of the late trumpet virtuoso Derek Watkins (1945-2013).

${ }^{1}$ M. Campbell, "Brass instruments as we know them today," Acta Acust. Acust. 90, 600-610 (2004).

${ }^{2}$ C. Gough, "Musical acoustics," in Springer Handbook of Acoustics, 1st ed. (Springer, New York, 2007), Chap. 15.

${ }^{3}$ S. Elliott and J. Bowsher, "Regeneration in brass wind instruments," J. Sound Vib. 83, 181-217 (1982).

${ }^{4}$ X. Pelorson, A. Hirschberg, R. van Hassel, A. Wijnands, and Y. Auregan, "Theoretical and experimental study of quasisteady-flow separation within the glottis during phonation: Application to a modified two-mass model," J. Acoust. Soc. Am. 96(6), 3416-3431 (1994).

${ }^{5} \mathrm{~N}$. Fletcher, "Autonomous vibration of simple pressure-controlled valves in gas flows," J. Acoust. Soc. Am. 93, 2172-2180 (1993).

${ }^{6} \mathrm{~S}$. Adachi and M. Sato, "Trumpet sound simulation using a twodimensional lip vibration model," J. Acoust. Soc. Am. 99(2), 1200-1209 (1996).

${ }^{7}$ D. Keefe, "Physical modeling of wind instruments," Comput. Music J. 16(4), 57-73 (1992).

${ }^{8}$ T. Helie, C. Vergez, J. Levine, and X. Rodet, "Inversion of a physical model of a trumpet," in Proceedings of the 38th IEEE Conference on Decision and Control (1999), Vol. 3, pp. 2593-2598. 
${ }^{9} \mathrm{C}$. Vergez and X. Rodet, "Dynamical systems and physical models of trumpet-like instruments: Analytical study and asymptotical properties," Acta Acust. Acust. 86, 147-162 (2000)

${ }^{10} \mathrm{C}$. Vergez and X. Rodet, "Trumpet and trumpet player: Model and simulation in a musical context," in ICMC'01, La Habana, Cuba (2001).

${ }^{11}$ A. Myers, R. W. Pyle, J. Gilbert, D. M. Campbell, J. P. Chick, and S. Logie, "Effects of nonlinear sound propagation on the characteristic timbres of brass instruments," J. Acoust. Soc. Am. 131, 678-688 (2012).

${ }^{12}$ W. Kausel, D. W. Zietlow, and T. R. Moore, "Influence of wall vibrations on the sound of brass wind instruments," J. Acoust. Soc. Am. 128, 3161-3174 (2010).

${ }^{13}$ S. Bilbao, "Time domain simulation of brass instruments," in 6th Forum Acusticum, Aalborg, Denmark (2011).

${ }^{14}$ B. d'Andrea Novel, J.-M. Coron, and T. Helie, "Asymptotic state observers for a simplified brass instrument model," Acta Acust. Acust. 96, 733-742 (2010).

${ }^{15}$ J. Kemp, M. van Walstijn, M. Campbell, J. Chick, and R. Smith, "Time domain wave separation using multiple microphones." J. Acoust. Soc. Am. 128(1), 195-205 (2010).

${ }^{16} \mathrm{G}$. de Sanctis and M. van Walstijn, "A frequency domain adaptive algorithm for wave separation," in 12th International Conference on Digital Audio Effects (DAFx-09), Como, Italy (2009).

${ }^{17}$ J. Kemp, S. Logie, J. Chick, R. Smith, and M. Campbell, "Analysis of transients for brass instruments under playing conditions using multiple microphones," in 10th Congrès Français d'Acoustique CFA'10, Lyon, France (2010).

${ }^{18}$ J. Chick, S. Logie, J. Kemp, M. Campbell, and R. Smith, "An exploration of extreme high notes in brass playing", in ISMA'10, Sydney, Australia (2010).
${ }^{19}$ S. Müller and P. Massarani, "Transfer-function measurement with sweeps" J. Audio Eng. Soc. 49(6), 443-471 (2001).

${ }^{20}$ S. Bilbao, Numerical Sound Synthesis (Wiley, Chichester, UK, 2009), Chap. 6.

${ }^{21} \mathrm{D}$. Keefe, "Acoustical wave propagation in cylindrical ducts: Transmission line parameter approximations for isothermal and nonisothermal boundary conditions," J. Acoust. Soc. Am. 75, 58-62 (1984).

${ }^{22}$ I. Podlubny, Fractional Differential Equations (Academic Press, San Diego, CA, 1999), Chap. 7.

${ }^{23} \mathrm{H}$. Levine and J. Schwinger, "On the radiation of sound from an unflanged circular pipe,” Phys. Rev. 73, 383-406 (1948).

${ }^{24}$ M. Atig, J.-P. Dalmont, and J. Gilbert, "Termination impedance of openended cylindrical tubes at high sound pressure level," C. R. Mec. 332, 299-304 (2004).

${ }^{25}$ L. R. Rabiner and R. W. Schafer, Digital Processing of Speech Signals (Prentice-Hall Englewood Cliffs, NJ, 1978), pp. 71-72.

${ }^{26}$ S. Stevenson, M. Campbell, S. Bromage, J. Chick, and J. Gilbert, "Motion of the lips of brass players during extremely loud playing," J. Acoust. Soc. Am. 125(4), EL152-EL157 (2009).

${ }^{27} \mathrm{~S}$. Stevenson, "Experimental investigations of lip motion in brass instrument playing," Ph.D. thesis, University of Edinburgh, UK, 2009.

${ }^{28}$ J. Saneyoshi, H. Teramura, and S. Yoshikawa, "Feedback oscillations in reed woodwind and brasswind instruments," Acta Acust. Acust. 62, 194-210 (1987).

${ }^{29}$ J. Kemp, "Theoretical and experimental study of wave propagation in brass musical instruments," Ph.D. thesis, University of Edinburgh, UK, 2002.

${ }^{30}$ J. Kemp and R. Smith, "Measuring the effect of the reflection of sound from the lips in brass musical instruments," in Acoustics 2012 Joint Meeting: 11th Congrès Français d'Acoustique and 2012 IOA annual meeting, Nantes, France (2012). 